\title{
Entrelacs
}

Cinéma et audiovisuel

Nouvelles formes audiovisuelles documentaires

\section{Webdocumentaire, documentaire interactif, idoc, jeu documentaire... Les enjeux des « nouvelles » formes audiovisuelles documentaires}

\section{Claire Chatelet}

\section{(2) OpenEdition \\ Journals}

Electronic version

URL: http://journals.openedition.org/entrelacs/1704

DOI: 10.4000/entrelacs. 1704

ISSN: 2261-5482

Publisher

Éditions Téraèdre

\section{Electronic reference}

Claire Chatelet, « Webdocumentaire, documentaire interactif, idoc, jeu documentaire... Les enjeux des « nouvelles » formes audiovisuelles documentaires », Entrelacs [Online], 12 | 2016, Online since 12

January 2016, connection on 10 December 2020. URL : http://journals.openedition.org/entrelacs/ 1704 ; DOI : https://doi.org/10.4000/entrelacs.1704

This text was automatically generated on 10 December 2020.

Tous droits réservés 


\title{
Webdocumentaire, documentaire interactif, idoc, jeu documentaire... Les enjeux des « nouvelles » formes audiovisuelles documentaires
}

\author{
Claire Chatelet
}

1 Pour son douzième numéro, Entrelacs, revue scientifique éditée par l'École Supérieure d'Audiovisuel et le Laboratoire de Recherche en Audiovisuel de l'Université Toulouse Jean-Jaurès (L.A.R.A.), propose de mettre en œuvre une réflexion sur les enjeux des «nouvelles» formes audiovisuelles documentaires, à l'heure des écrans connectés (ordinateurs, tablettes tactiles, écrans hybrides, smartphones). Que reste-t-il du film, quand ses formes, soudain ouvertes, délinéarisées, variables, se déploient à partir d'une interface, selon un "graphe de navigation ${ }^{1}$ » qui sollicite et engage des «spectateurs actants $^{2}$ »? Comment «documenter le réel» quand l'objet audiovisuel devient modulaire, manipulable, «actable ${ }^{3}$ »; quand l'accès à l'objet même offre de multiples entrées et propose autant de prolongements? Comment envisager les modalités de la $\mathrm{r}$ éception, quand les spectateurs s'informent en utilisateurs, en joueurs, voire en « cocréateurs»?

Dès 2002, à l'occasion d'un cycle intitulé les ciném@s de demain, le Centre Pompidou s'interrogeait sur une forme audiovisuelle émergente qualifiée de " web documentaire » et se proposait même de la définir, en ces termes : " un documentaire travaillé avec les outils multimédia, textes, images, vidéos, une manière de mettre les nouvelles technologies au service de la connaissance et d'un point de vue ${ }^{4} »$. Si les initiateurs de la manifestation insistèrent sur la variété de ses formes, ils appréhendèrent néanmoins déjà le «web documentaire » en tant que "genre encore peu exploité, parce que (...) mal défini (..) aux frontières de la webtv, du magazine en ligne ou du journal de bord ${ }^{5}$ ».

3 Si quelques expériences audiovisuelles hybrides ont émergé sur le web au tournant des années $2000^{6}$, la production de formes documentaires « connectées " s'est réellement 
développée en France autour des années 2008-2010, avec des réalisations marquantes comme Voyage au bout du charbon de Samuel Bollendorf (2008), Gaza/Sderot (2008), Prison Valley de David Dufresne (2010) ou encore Brèves de trottoirs d'olivier Lambert et Thomas Salva (2010). Dans un même élan, des sociétés de production spécialisées dans les nouvelles écritures audiovisuelles ont vu le jour, (Honkytonk, Narrative, Darjeeling, Hans Lucas, Ligne $4 . ..)^{7}$, tout comme des services dédiés dans les grands groupes audiovisuels publics (Arte Pôle Web, Département des Nouvelles Ecritures de France Télévisions). A l'instar de son rôle de soutien à la production cinématographique, le CNC a également favorisé le développement de ces «nouvelles » formes en ouvrant en 2007 un fonds d'aide aux projets « nouveaux médias » afin d'accompagner " les œuvres audiovisuelles innovantes qui intègrent les spécificités de l'Internet et/ou des écrans mobiles dans leur démarche artistique et de diffusion ${ }^{8} »$.

D'un point de vue pratique, la réalisation de ces « œuvres » nécessite la collaboration de compétences et métiers éloignés du secteur cinématographique : développeur, webdesigner, web architect, community manager... De plus, il apparait que la majorité d'entre elles sont initiées par des journalistes, des photo-journalistes ou des photographes. C'est peut-être ce qui explique une certaine méfiance du milieu cinématographique envers ces formes, voire même un rejet total de la part de certains cinéastes, comme en témoigne par exemple cette critique du documentariste Rémi Lainé dans la Lettre de la Société Civile des Auteurs Multimédias de février 2010 : " Le documentaire n'a-t-il pas vocation à embarquer son auditoire dans une histoire, lui faire oublier le temps d'un film qu'il est devant un écran (...). Sur le net, nous explique$\mathrm{t}$-on, il convient de "délinéariser" le récit. (...) Ce que l'on découvre reste très consensuel et l'invitation au clic n'y change rien. Jamais la forme ne débride le propos. La réflexion semble s'arrêter à l'habillage. Sur le fond, rien qui n'ait déjà été vu et revu à la télé $(. . .)^{9}$ ».

Qu'en est-il aujourd'hui, alors que pullulent les propositions audiovisuelles documentaires sur le web ? Peut-on véritablement parler de genre, voire de sous-genre, sinon de format? Y a-t-il des critères distinctifs communs à ces projets?

6 L'hétérogénéité des formes documentaires connectées, qui se manifeste dans l'instabilité des termes pour les qualifier (documentaire interactif, webdoc, webdocumentaire, idoc, jeu documentaire, expérience documentaire interactive...) semble rendre caduque toute tentative de stricte dénomination. Cependant, au-delà de ce flou terminologique, un terme principal perdure, celui de "documentaire ", accolé à un critère technologique qui a trait, soit au médium/à la spécificité du canal de diffusion (le web), soit à la qualité de la relation entre le récepteur et le programme (interactivité, jouabilité ${ }^{10}$ ). La référence au genre cinématographique (documentaire), comme si elle était garante d'une certaine légitimité, paraît donc essentielle aussi bien pour les concepteurs de ces formes que pour leurs commentateurs ; pourtant hormis quelques expérimentateurs, peu de cinéastes les ont jusque-là réellement expérimentées ${ }^{11}$.

7 Ce numéro d'Entrelacs vise précisément à interroger ces « nouvelles » formes au regard du cinéma d'une façon générale, et du documentaire en particulier, tant du point de vue de la création, que de la réception. La première partie s'attache aux caractéristiques techniques et formelles des webdocumentaires et aux relations spectatorielles spécifiques qu'ils déterminent ; la deuxième partie interroge le 
renouvellement des genres et des procédés narratifs, la troisième partie enfin, propose l'analyse de quelques œuvres interactives singulières.

\section{NOTES}

1. Selon les termes de Jean-Louis Weissberg (Présence à distance : Déplacement virtuel et réseaux numériques : pourquoi nous ne croyons plus à la télévision, L'Harmattan, 2000).

2. Pour reprendre la formule de Marida Di Crosta (Entre cinéma et jeux vidéo : l'interfacefilm, Bruxelles/ Paris, De Boeck/Ina, 2009). Jean-Louis Weissberg proposa dès 2000, le concept largement repris de « spectacteur ».

3. Marida di Crosta propose les terminologies de «films actables » et d' « interface-film » en expliquant : «Interface-film est pour moi un synonyme moins équivoque de film interactif. Cela désigne moins un genre qu'une configuration langagière spécifique à ces objets hybrides, à mi-chemin entre le film et autre chose » (ibid., p.8). Quant à Gwenola Wagon, elle envisage un " cinéma jouable » (Thèse de doctorat, Utopies d'un cinéma interactif Accessibilité des images en mouvement, sous la direction de Jean-Louis Boissier, Université Paris 8, 2006).

4. C.f. http://www.centrepompidou.fr/cpv/resource/cibK7r/rAd9B4

5. Ibid.

6. Car l'évolution de l'internet les ont rendu techniquement possibles. C.f La Cité des mortes (2005), Thanatorama (2007) produites par Upian.

7. Upian, l'une des société de productions les plus actives existe depuis 1998, spécialisée au départ dans la création et le développement de sites Web publicitaires, événementiels et corporate, ainsi que la conception et le développement jeux en ligne.

8. C.f : http://www.cnc.fr/web/fr/aide-aux-projets-nouveaux-medias

9. c.f : «Ecran de fumée » de Rémi Lainé (p.9). Disponible sur : http://www.scam.fr/ Portals/0/Contenus/ documents/lettres/BAT37_lettre_Scam.pdf

10. Interactivité qui apparaît de façon plus implicite il est vrai, dans la référence aux modalités de médiation des jeux-vidéo dans le terme " jeu documentaire " proposé notamment par les concepteurs de Fort Mc Money (2013).

11. Beaucoup d'expérimentatrices on pense à La vie à sac de Sólveig Anspach (2010) pour Médecins du Monde à l'occasion des trente ans de l'association, Laeticia Masson avec The End, etc... (2013), Claire Simon avec la version interactive de Gare du Nord, ou encore Yaël André avec Synaps (2014). Dans ces deux derniers cas il s'agit d'un projet qui accompagne un film linéaire pour le cinéma ou la télévision. Quelques expérimentateurs aussi comme le cinéaste franco-marocain Nabil Ayouch avec To My Land (2014) sorte de journal de bord " interactif " poursuivant son film documentaire My Land sorti en 2013, Ritty Panh avec le projet participatif One Dollar (2014), Vincent Ravalec avec Zero G : un vol sans gravité (2013), Lech Kowalski avec Cuts (2012). 
INDEX

Mots-clés: Webdocumentaire, documentaire interactif, idoc, jeu documentaire, transmedia

\section{AUTHOR}

\section{CLAIRE CHATELET}

MCF en audiovisuel et nouveaux médias, Université Paul-Valéry, Montpellier, Membre du RIRRA21 